\title{
A Generalization of the Barnes $G$-Function
}

\author{
R. SCHUSTER
}

\begin{abstract}
Generalizing the Barnes $G$-function we define an entire function of order $m$ with the zeros $k$ with multiplicity $k^{m}(k, m \in N)$. We prove functional equations for it and study its asymptotic behaviour and Taylor series. This generalization is useful in order to describe the topological zeros of the Selberg zeta function with respect to the spectrum of the Laplace operator for differential $p$-forms on $n$-dimensional compact hyperbolic space forms.
\end{abstract}

Key words: Barnes $G$-function, hyperbolic space form, zeta function, spectral theory

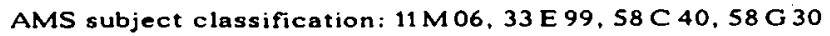

\section{Introduction}

The Selberg zeta function with respect to the spectrum of the Laplace operator for differential $p$-forms on $n$-dimensional compact hyperbolic space forms plays an important role for the study of the length spectrum and eigenvalue spectra. One part of the zeros and poles of the Selberg zeta function is related to topological properties of the space form, the other one is related to the eigenvalue spectrum of the Laplace operator. We want to give a generalization of the Barnes $G$-function in order to handle the topological aspects. Voros [14] used the Barnes $G$-function to discuss the two-dimensional case.

First we want to give some details about the spectral geometric background of the intended generalization. Let $V$ be an $n$-dimensional compact hyperbolic space form and let $\Omega$ be the set of non-trivial free homotopy classes of $V$. In every class $\omega \in \Omega$ there lies exactly one geodesic line. We denote by $l(\omega)$ and $v(\omega)$ its lenght and multiplicity, respectively. The parallel displacement along the closed geodesic line induces an isometry of the tangent space in every point of the geodesic line with the eigenvalues $\beta_{1}(\omega), \ldots, \beta_{n-1}(\omega), 1$ with $\left|\beta_{j}(\omega)\right|=1(i=$ $1, \ldots, n-1)$. Let $e_{p}(\omega)$ be the $p^{\text {th }}$ elementary symmetric function of the $\beta_{j}(\omega)$, and put $e_{0}(\omega)=1$. Further on, we introduce the weight

$$
\sigma(\omega)=\frac{1}{v(\omega)} e^{N I(\omega)} \prod_{j=1}^{-1} \frac{1}{e^{I(\omega)}-\beta(\omega)} \text { with } N=\frac{n-1}{2} .
$$

Let $S_{p}$ denote the $p$-spectrum of the Laplace operator $\Delta=d \delta+\delta d$. Thereby we have used the differential operator $d$ and the codifferential operator $\delta$ acting on differential $p$-forms on $V$. It is well known that the Selberg zeta function for the classical case $n=2$ and $p=0$ that is defined by

$$
2(s)=\prod_{\omega \in \Omega, v(\omega)=1} \prod_{k=0}^{\infty}\left(1-e^{-I(\omega)(s+k)}\right) \text { for } \operatorname{Re} s>1,
$$

bears a very striking resemblance to the Riemann zeta function (as well as differences). One 
easily shows

$$
\sum_{\omega \in \Omega} I(\omega) \sigma(\omega) e^{-I(\omega)(s-1 / 2)}=\frac{d \ln 2(s)}{d s} .
$$

There are many papers about the two-dimensional case, for further information we refer to [6].

We define

$$
\Psi_{p}(s)=\Gamma(p+1) \Gamma(n-p) \sum_{\omega \in \Pi}(\omega) \sigma(\omega) e_{p}(\omega) e^{-l(\omega)(s-N)} \text { for } n \text { even, } \operatorname{Re} s>2 N, N=\frac{n-1}{2} .
$$

This function $\Psi_{p}$ can be considered as the logarithmic derivative of the Selberg zeta function (cf. [11]) and it has a meromorphic continuation to the whole complex plane. One part of the poles of $\Psi_{p}$ is related to the eigenvalue spectrum $S_{p}$ of the space form $V$, and the other one is related to topological properties (with the exception of the points $N$ and $N \pm|N-p|$ in which the residues of the poles of $\Psi_{p}$ may reflect both topological and spectral aspects of $V$ ). There are the (topological) poles $s=-k(k \in \mathbb{N})$ of the function $\Psi_{p}$ with residues $(-1)^{n / 2} 2(N+k) \times(V)$ $\times P\left(-(N+k)^{2}\right)$ for $n$ even (cf. [11]). Thereby $\chi$ denotes the Euler-Poincaré characteristic of $V$, and the polynomial $P$ is defined by $P(s)=\prod_{u=1 / 2, u \neq|N-p|}^{N}\left(s+u^{2}\right)$. In order to generalize the factorization of the Selberg zeta function given by Voros [14] for $n=2$, we look for a function having the zeros $s=-k(k \in N)$ with multiplicities $k^{m}(m \in N)$. For further information about Selberg zeta functions and the spectral geometric background we refer to $[2-6,10,12,14]$. Our main result is the following

Theorem A: The generalized Barnes $G$-function $G_{m}$ defined by

$$
\begin{aligned}
G_{m}(1+z)= & \exp \left\{-\sum_{j=1}^{m} \frac{\zeta(j-m)}{j}(-z)^{j}-\left(\left(\gamma+\sum_{r=1}^{m} \frac{1}{r}\right) /(m+1)\right)(-z)^{1+m}+\sum_{j=1}^{m}\left({ }_{j}^{m}\right) \zeta^{\prime}(j-m)(-z)^{j}\right\} \\
& \times \prod_{k=1}^{\infty}\left\{\left(1+\frac{z}{k}\right)^{k^{m}} \exp \left(\left(\sum_{r=1}^{m+1} \frac{1}{r} \frac{1}{k^{r}}(-z)^{r}\right) k^{m}\right)\right\} \text { for } m=0,1,2, \ldots
\end{aligned}
$$

by help of the Riemann zeta function $\zeta$ and the Euler number $\gamma$ is an entire function of order $m$ with the zeros $-k$ with multiplicity $k^{m}(k \in \mathbb{N})$. Further on, there is the zero 0 with multiplicity 1 for $m=0$. The used infinite product is absolutely convergent. We have $G_{m}(1)=1$, and $G_{m}$ satisfies the functional equation

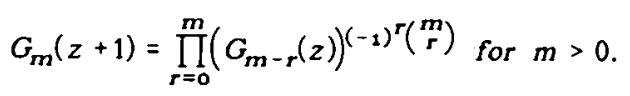

Proposition 5 will describe the asymptotic behaviour of $G_{m}(z+1)$ for $z \rightarrow \infty$ and Proposition 6 will give the Taylor series of $\ln G_{m}(z+1)$ for $|z|<1$. The function $G_{m}$ generalizes also the gamma function. In fact, we have $G_{0}=1 / \Gamma$.

\section{Spectral formalism}

We want to apply techniques described in [14] in order to handle abstract spectral sequences. For further information cf. [7 - 9]. For the convenience of the reader, we recall those formulas of [14] which we will use later on.

One studies an abstract sequence $\left(\lambda_{k}\right)_{k=0}^{\infty} \subset \mathbb{R}$ with $0<\lambda_{0}, \lambda_{k} \leq \lambda_{k+1}$ for all $k$ and $\lambda_{k} \rightarrow \infty$ for $k \rightarrow \infty$. It is supposed that the partition function $\omega(t)=\sum_{k=0}^{\infty} \exp \left(-\lambda_{k} t\right)$ converges for all $t$ 
with $\operatorname{Re} t>0$ and admits a full asymptotic expansion

$$
\omega(t) \sim \sum_{k=0}^{\infty} c_{i_{k}} t^{i_{k}} \text { for } t \rightarrow+0
$$

for a suitable increasing sequence $\left(i_{k}\right)$ of real exponents with $i_{k} \rightarrow \infty$ for $k \rightarrow \infty$ and $i_{0}<0$. The number $\mu=-i_{0}$ is called the order of the sequence $\left(\lambda_{k}\right)$. With respect to our applications, we suppose the order $\mu$ to be an integer. It will be useful to define, by convention, $c_{\alpha}=0$ for any real number $\alpha$ which is not in the sequence $\left(i_{k}\right)$. We now define a zeta function $Z$ by

$$
Z(s)=\sum_{k=0}^{\infty} \lambda_{k}^{-s} \text { for } \operatorname{Re} s>\mu .
$$

We emphasize that this zeta function is quite different from the Selberg zeta function. It has a meromorphic continuation to the whole complex plane, it is holomorphic for $\operatorname{Re} s>\mu$ and has the simple poles $s=-i_{k}$ with the residues $c_{i_{k}} / \Gamma\left(i_{k}\right)$. It is convenient to introduce a two-parametric zeta function by $Z(s, a)=Z^{*}(s)$, where $Z^{*}$ is the zeta function related to the global shift $\lambda_{k} \rightarrow \lambda_{k}+a$ of the sequence $\left(\lambda_{k}\right)$. The Weierstrass product $\Delta$ as a regularized Fredholm determinant is defined by

$$
\Delta(\lambda)=\prod_{k=0}^{\infty}\left(1-\frac{\lambda}{\lambda_{k}}\right) \exp \left(\sum_{r=1}^{\mu} \frac{\lambda^{r}}{r \lambda_{k}^{r}}\right) .
$$

This is the unique entire function of order $\mu$ having the zeros $\lambda_{k}$ which satisfies the normalization conditions

$$
\ln \Delta(0)=\left.\frac{d \ln \Delta(\lambda)}{d \lambda}\right|_{\lambda=0}=\ldots=\left.\frac{d^{\mu} \ln \Delta(\lambda)}{d \lambda^{\mu}}\right|_{\lambda=0}=0 .
$$

We get the convergent series

$$
R(\lambda)=\sum_{k=0}^{\infty} \mu !\left(\lambda_{k}-\lambda\right)^{-\mu-1}=\frac{-d^{\mu+1} \ln \Delta(\lambda)}{d \lambda^{\mu+1}} \text { for } \lambda<0
$$

and

$$
\frac{d^{\mu+j} \ln \Delta(\lambda)}{d \lambda^{\mu+j}}=-(\mu+j-1) ! \sum_{k=0}^{\infty}\left(\lambda_{k}-\lambda\right)^{-\mu-j} \quad \text { for } \lambda<0 \text { and } j \in N
$$

The functional determinant $D$ is introduced by $D(\lambda)=\exp \left(-Z^{\prime}(0,-\lambda)\right)$, the sign thereby denotes the derivative with respect to the first variable. We use the Finite Part prescription as usual:

$$
\operatorname{FPf}(s)= \begin{cases}f(s) & \text { if } s \text { is not a pole } \\ \lim _{\varepsilon \rightarrow 0}(f(s+\varepsilon)-\text { residue } / \varepsilon) & \text { if } s \text { is a simple pole } .\end{cases}
$$

There is an important relation between the Weierstrass product and the functional determinant:

$$
\left.D(\lambda)=\exp \left\{-Z^{\prime}(0)-\sum_{j=1}^{\mu} \mathrm{FP} \frac{Z(j)}{j} \lambda^{j}-\sum_{j=2}^{\mu} c_{-j}\left(\left(\sum_{r=1}^{j-1} \frac{1}{r}\right) / j !\right) \lambda^{j}\right)\right\} \Delta(\lambda) .
$$

One has

$$
\frac{d^{\mu+1}(-\ln D(\lambda))}{d \lambda^{\mu+1}}=\frac{d^{\mu+1}(-\ln \Delta(\lambda))}{d \lambda^{\mu+1}}=R(\lambda) .
$$

The normalization conditions (3) are related to the normalization point $\lambda=0$ : 


$$
-\ln \Delta(\lambda)=\int_{0}^{\lambda} \int_{0}^{v_{1}} \ldots \int_{0}^{v_{\mu}} R(\mu) d \mu \ldots d \nu_{1} d \lambda .
$$

If we consider asymptotic expansions, it is useful to choose $-\infty$ instead of 0 as the lower integration point, and we have

$$
-\ln D(\lambda)=\int_{-\infty}^{\lambda} \int_{-\infty}^{\psi_{1}} \ldots \int_{-\infty}^{\nu_{\mu}} R(\mu) d \mu \ldots d v_{1} d \lambda .
$$

We denote the functional determinant $D(\lambda)$ under a global shift $\lambda_{k} \rightarrow \lambda_{k}+a$ by $D_{a}(\lambda)$. Then we get $D_{a}(\lambda)=D(\lambda-a)$. The analogous transformation of $\Delta(\lambda)$ is much more complicated. Further on, we get the asymptotic expansion

$$
\ln D(\lambda) \sim-\sum_{0=-i_{k}} c_{i_{k}} \Gamma\left(i_{k}\right)(-\lambda)^{-i k_{k}}+\sum_{j=0}^{\mu} c_{-j}\left(\left(\ln (-\lambda)-\sum_{r=1}^{j} r^{-1}\right) / j !\right) \lambda^{j} \text { for } \lambda \rightarrow-\infty .
$$

\section{The generalization of the Barnes $G$-function}

The Barnes $G$-function $G=G(z)$ is defined by the infinite product

$$
G(z+1)=(2 \pi)^{z / 2} e^{-\left(z+(1+\gamma) z^{2}\right) / 2} \prod_{k=1}^{\infty}\left(\left(1+\frac{z}{k}\right)^{k} e^{-z+z^{2} / 2 k}\right)
$$

with the Euler number $\gamma$. It is an entire function of order 2 . Its zeros are the negative integers $-k$ with multiplicity $k(k \in \mathbb{N})$. The Barnes $G$-function satisfies the normalization condition $G(1)=1$ and the functional equation $G(z+1)=\Gamma(z) G(z)$ with the gamma function $\Gamma$. The functions $D$ or $\Delta$ with respect to the sequence of integers $k(k \in \mathbb{N})$ with multiplicity $k$ don't satisfy the mentioned normalization condition and functional equation. Thus we have to handle the problem to find useful normalization conditions for our spectral functions. We put $G_{1}=G$ and $G_{0}=1 / \Gamma$. The function $G_{0}$ is an entire function of order one, its zeros being the negative integers $-k$ and zero, each with multiplicity 1 , and it satisfies the normalization condition $G_{0}(1)=1$.

Now we will introduce a generalized Barnes $G$-function $G_{m}(m \in \mathrm{N})$ which satisfies the following conditions:

(G) $G_{2} G_{m}$ is an entire function of order $m$ with the zeros $-k(k \in \mathbb{N})$ with multiplicity $k^{m}$.

(G) $G_{m}(1)=1$.

(G) We have the functional equation $G_{m}(z+1)=\prod_{r=0}^{m}\left(G_{m-r}(z)\right)^{(-1)^{r}\left(\begin{array}{c}m \\ r\end{array}\right)}$.

We apply the described spectral formalism to the sequence of integers $k$ with multiplicity $k^{m}$ (we will call this sequence $F_{m}$ ). We get the partition function

$$
\omega_{m}(t)=\sum_{k=1}^{\infty} k^{m} \frac{1}{e^{k t}}=\left(-\frac{d}{d t}\right)^{m} \frac{1}{e^{t}-1} .
$$

It is well known (cf. $[1,13])$ that

$$
\frac{1}{e^{\ell}-1}=-\frac{1}{2}+\frac{1}{t}+\sum_{r=1}^{\infty} \frac{(-1)^{r-1}}{(2 r) !} B_{r} t^{2 r-1}
$$

with the Bernoulli numbers $B_{r}\left(B_{1}=1 / 6, B_{2}=1 / 30, B_{3}=1 / 42, B_{4}=1 / 30, \ldots\right)$. It follows that the sequence $F_{m}$ is of order $\mu=m+1$. We get 


$$
\left(-\frac{d}{d t}\right)^{m} \frac{1}{e^{t}-1}=m ! \frac{1}{t^{m+1}}+\sum_{r=\delta_{0}}^{\infty}(-1)^{r-1-m} \frac{(2 r-1-m) !}{2 r} B_{r} t^{2 r-1-m}
$$

with $r_{0}=(m+1) / 2$ for odd $m$ and $r_{0}=(m+2) / 2$ for even $m>0$. If we denote the coefficients $c_{i_{k}}$ of the asymptotic expansion (1) with respect to the sequence $F_{i n}$ by $c_{i_{k}}^{(m)}$, we conclude

$$
c_{0}^{(0)}=-\frac{1}{2}, c_{-(m+1)}^{(m)}=m !, c_{2 r-1-m}^{(m)}=(-1)^{r-1+m} \frac{(2 r-1-m) !}{2 r} B_{r} \text { for } r \in Z, r \geq r_{0} .
$$

It valids $c_{\alpha}^{(m)}=0$ if and only if $c_{\alpha}^{(m)}$ is not included above. So we have enough information about the asymptotic expansion (1). The zeta function $Z_{m}$ defined by (2) with respect to $F_{m}$ is given by

$$
Z_{m}(s)=\sum_{k=1}^{\infty} k^{m} k^{-s}=\zeta(s-m) .
$$

Thereby $\zeta$ denotes the Riemann zeta function. In the first part of this paper we have mentioned the analogies between the Selberg zeta function and the Riemann zeta function. It turns out that the Riemann zeta function also plays an important role for the description of those zeros of the Selberg zeta function which reflect topological properties of the related hyperbolic space form $V$ (which are only depending on $p, n$ and $\chi(V)$ ). We immediately get the following

Proposition 1: The regularized Fredholm determinant $\Delta_{m}$ with respect to the sequence $F_{m}$ is given by

$$
\Delta_{m}(\lambda)=\prod_{k=1}^{\infty}\left(1-\frac{\lambda}{k}\right)^{k^{m}} \exp \left(\left(\sum_{r=1}^{m+1} \frac{1}{r k^{r}} \lambda^{r}\right) k^{m}\right)
$$

In order to describe the relation (6) between the regularized Fredholm determinant $\Delta_{m}$ and the related functional determinant $D_{m}$ we have to consider the relation

$$
\begin{aligned}
& -Z_{m}^{\prime}(0)-\sum_{j=1}^{\mu} \frac{\mathrm{FP} Z_{m}(j)}{j} \lambda^{j}-\sum_{j=2}^{\mu} c_{-j}\left(\left(\sum_{r=1}^{j-1} \frac{1}{r}\right) / j !\right) \lambda^{j} \\
& =-\zeta(-m)-\sum_{j=1}^{m+1} \frac{\mathrm{FP} \zeta(j-m)}{j} \lambda^{j}-\left(\left(\sum_{r=1}^{m} \frac{1}{r}\right) /(m+1)\right) \lambda^{m+1} \\
& =-\zeta^{\prime}(-m)-\sum_{j=1}^{m} \frac{\zeta(j-m)}{j} \lambda^{j}-\left(\left(\gamma+\sum_{r=1}^{m} \frac{1}{r}\right) /(m+1)\right) \lambda^{m+1} .
\end{aligned}
$$

Thereby we have used FP $\zeta(1)=\gamma$ (Euler number). Notice further that (cf. [13]) $\zeta(0)=-1 / 2$, $\zeta(-2 r)=0, \zeta(1-2 r)=(-1)^{r} B_{r} / 2 r(r \in \mathbb{N})$. It is immediately clear that both functions $D_{m}(\lambda$ -1) and $\prod_{r=0}^{m}\left(D_{m-r}(\lambda)\right)^{(-1)^{r}\left(\begin{array}{c}m \\ r\end{array}\right)}$ are entire functions of order $\mu=m+1$ having the zeros $\lambda=k$ with multiplicities $(k-1)^{m}(k \in \mathbb{N})$. Using (4) and (7) we get

$$
\begin{aligned}
-\left(\frac{d}{d \lambda}\right)^{m+2} \ln D_{m}(\lambda-1) & =(m+1) ! \sum_{k=1}^{\infty} k^{m} \frac{1}{(k-\lambda+1)^{m+2}} \\
& =(m+1) ! \sum_{k=1}^{\infty}(k-1)^{m} \frac{1}{(k-\lambda)^{m+2}}
\end{aligned}
$$




$$
\begin{aligned}
& =(m+1) ! \sum_{r=0}^{m}(-1)^{r}\left(\begin{array}{c}
m \\
r
\end{array}\right) \sum_{k=1}^{\infty} k^{m-r} \frac{1}{(k-\lambda)^{m+2}} \\
& =\sum_{r=0}^{m}(-1)^{r}\left(\begin{array}{c}
m \\
r
\end{array}\right)\left(\frac{d}{d \lambda}\right)^{r}(m+1-r) ! \sum_{k=1}^{\infty} k^{m-r} \frac{1}{(k-\lambda)^{m+2-r}} \\
& =-\left(\frac{d}{d \lambda}\right)^{m+2} \sum_{r=0}^{m}(-1)^{r}\left(\begin{array}{c}
m \\
r
\end{array}\right) \ln D_{m-r}(\lambda)
\end{aligned}
$$

for $\lambda<0$. Further on, an easy calculation shows that

$$
\lim _{\lambda \rightarrow-\infty}\left(\frac{d}{d \lambda}\right)^{j} \ln D_{m}(\lambda-1)=0=\lim _{\lambda \rightarrow-\infty}\left(\frac{d}{d \lambda}\right)^{j} \sum_{r=0}^{m}(-1)^{r}\left(\begin{array}{c}
m \\
r
\end{array}\right) \ln D_{m-r}(\lambda) \text { for } j=0,1,2, \ldots, m-1 .
$$

By using the normalization point $\lambda=-\infty$, it follows

Proposition 2: The functional determinant $D_{m}$ with respect to the spectral sequence $F_{m}$ satisfies the functional equation

$$
D_{m}(\lambda-1)=\prod_{r=0}^{m}\left(D_{m-r}(\lambda)\right)^{(-1)^{r}\left(\begin{array}{r}
m \\
r
\end{array}\right)} .
$$

The normalization point $\lambda=-\infty$ turns out to be essential to prove the functional equation for the generalized Barnes $G$-function. Indeed, we define

$$
D_{m}(\lambda)=\exp \left(\sum_{r=0}^{m} p_{r}^{m} \lambda^{r}\right) G_{m}(1-\lambda)
$$

and specify the constants $p_{r}^{m}$ by the conditions $(G)_{2}$ and $(G)_{3}$. Using $\lambda=0$ in (14), $(G)_{2}$ implies $D_{m}(0)=\exp p_{\mathrm{o}}^{m}$. Now we take into consideration that we get $D_{m}(0)=\exp (-\zeta(-m))$ by (6),(11) and (12). It follows

$$
p_{0}^{m}=-\zeta(-m) \text {. }
$$

Using (13) and (14) we see that $(G)_{3}$ is satisfied if and only if

$$
\sum_{r=1}^{m} p_{r}^{m}\left((\lambda-1)^{r}-\lambda^{r}\right)=\sum_{r=1}^{m}(-1)^{r}\left(\begin{array}{c}
m \\
r
\end{array}\right)\left(\sum_{j=0}^{m-r} p_{j}^{m-r} \lambda^{j}\right)
$$

Proposition 3: Supposing (15) the equations (16) have the unique solution $p_{k}^{m}=-\left(\begin{array}{c}m \\ k\end{array}\right) \zeta(k-m)$.

Proof: If we compare the coefficients of $\lambda^{k}$ in (16), we get a system of linear equations for $p_{1}^{m}, \ldots, p_{m}^{m}$ with a triangle matrix and elements different from zero on its diagonal. Thus $p_{1}^{m}, \ldots, p_{m}^{m}$ are uniquely determined. Indeed, we get the equations

$$
\sum_{r=1}^{m-k+1}(-1)^{r}\left(\begin{array}{c}
k+r-1 \\
r
\end{array}\right) p_{k+r-1}^{m}=\sum_{r=1}^{m-k+1}(-1)^{r}\left(\begin{array}{c}
m \\
r
\end{array}\right) p_{k-1}^{m-r}
$$

for the coefficients of $\lambda^{k+1}(k=1,2, \ldots, m)$. But these equations are identities if we use $p_{k}^{m}=$ $-\left(\begin{array}{c}m \\ k\end{array}\right) \zeta^{\prime}(k-m)$ and $(k+r-1)(k+r-1)=\left(\begin{array}{c}m \\ r\end{array}\right)\left(\begin{array}{c}m-r \\ k-1\end{array}\right)$

Proposition 4: The generalized Bames $G$-function $G_{m}$ defined by (14) can also be introduced by the infinite product definition 


$$
\begin{aligned}
G_{m}(z+1)= & \exp \left\{-\zeta^{\prime}(-m)-\sum_{j=1}^{m} \frac{\zeta(j-m)}{j}(-z)^{j}-\left(\left(\gamma+\sum_{r=1}^{m} \frac{1}{r}\right) /(m+1)\right)(-z)^{m+1}+\sum_{j=0}^{m}\left(\begin{array}{l}
m \\
j
\end{array}\right) \zeta^{\prime}(j-m)(-z)^{j}\right\} \\
& \times \prod_{k=1}^{\infty}\left\{\left(1+\frac{z}{k}\right)^{k^{m}} \exp \left(\left(\sum_{r=1}^{m+1} \frac{1}{r k^{r}}(-z)^{r}\right) k^{m}\right)\right\} .
\end{aligned}
$$

Proof: In order to get the infinite product definition of $G_{m}$, we have to consider equation (6) with respect to our situation. Using (11),(12), (14) and Proposition 3, we get the assertion

Theorem $A$ is an immediate consequence of the results above. We remark that the Riemann zeta function plays an important role for the normalization condition $(G)_{3}$. Using (10) and (14), the calculated coefficients $c_{\alpha}^{(m)}$ and Proposition 3, we get

Proposition 5: The generalized Barnes $G$-function $G_{m}$ has the following asymptotic behaviour:

(i) For odd $m$,

$$
\ln G_{m}(1+z) \sim\left\{\begin{array}{l}
\sum_{j=0}^{m}\left(\begin{array}{c}
m \\
j
\end{array}\right) \zeta(j-m)(-z)^{j}+\sum_{r=(m+3) / 2}^{\infty} \frac{(-1)^{r+m}}{2 r(2 r-1-m)} B_{r} z^{m+1-2 r} \\
+\frac{1}{m+1}\left(\ln z-\sum_{r=1}^{m+1} \frac{1}{r}\right)(-z)^{m+1}+\frac{(-1)^{(m+1) / 2}}{m+1} B_{(m+1) / 2} \ln z
\end{array}\right.
$$

(ii) For even $m>0$,

$$
\ln G_{m}(1+z) \sim\left\{\begin{array}{l}
\sum_{j=0}^{m}\left(\begin{array}{l}
m \\
j
\end{array}\right) \zeta(j-m)(-z)^{j}+\sum_{r=m / 2+1}^{\infty} \frac{(-1)^{r+m}}{2 r(2 r-1-m)} B_{r} z^{m+1-2 r} \\
+\frac{1}{m+1}\left(\ln z-\sum_{r=1}^{m+1} \frac{1}{r}\right)(-z)^{m+1}
\end{array}\right.
$$

(iii) For $m=0$,

$$
\ln G_{0}(1+z) \sim \zeta^{\prime}(0)+\sum_{r=1}^{\infty} \frac{(-1)^{r}}{2 r(2 r-1)} B_{r} z^{1-2 r}-\frac{1}{2} \ln z+(1-\ln z) z \text { for } z>0, z \rightarrow \infty .
$$

Next we will give the Taylor series of $\ln G_{m}(1+z)$ for $|z|<1$. By (5) we have

$$
\left.\left(\frac{d}{d \lambda}\right)^{\mu+j} \ln \Delta_{m}(\lambda)\right|_{\lambda=0}=-(\mu+j-1) ! \sum_{k=1}^{\infty} k^{-\mu-j+m}=-(m+j) ! \sum_{k=1}^{\infty} k^{-j-1}=-(m+j) ! \zeta(j+1)
$$

for $j \in \mathbb{N}$. On the other hand, the normalization point $\lambda=0$ in equation (8) implies $\ln \Delta_{m}(\lambda)=$ $O\left(\lambda^{2+m}\right)$ for $\lambda \rightarrow 0$. Applying (6), (12) and (14) we get

Proposition 6: The generalized Barnes $G$-function $G_{m}$ has the Taylor series

$$
\begin{aligned}
\ln G_{m}(1+z)= & -\sum_{j=1}^{m} \frac{\zeta(j-m)}{j}(-z)^{j}-\left(\left(\gamma+\sum_{r=1}^{m} \frac{1}{r}\right) /(m+1)\right)(-z)^{m+1} \\
& +\sum_{j=1}^{m}\left(\begin{array}{c}
m \\
j
\end{array}\right) \zeta(j-m)(-z)^{j}-\sum_{j=1}^{\infty} \frac{\zeta(j+1)}{m+1+j}(-z)^{m+1+j}
\end{aligned}
$$

for $|z|<1$. 
Using the generalized Barnes $G$-function, we would be able to find a function having the numbers $-k$ as zeros with multiplicity $(N+k) P\left(-(N+k)^{2}\right)$ and the value 1 at the point $s=N$. We will give details about the application to the situation remarked in the first part of this paper in a subsequent paper.

\section{REFERENCES}

[1] Fichtenholz, G. M.: Differential- und Integralrechnung, Vol.ll. Berlin: Dt. Verlag Wiss. 1971.

[2] FRIED, D.: Analytic Torsion and closed geodesics on hyperbolic manifolds. Invent. Math. $84(1986), 523$ - 540.

[3] FRIED. D.: Zeta functions of Ruelle and Selberg.Ann. Sci.Ecole Norm. Sup. 19 (1986), 491 $-517$.

[4] GANGOLLI, R.: Asymptotic behaviour of spectra of compact quotients of certain spaces. Acta Math. 121 (1968), $151-192$.

[S] GANGOLLI, R.: Zeta function of Selberg's type for compact space forms of symmetric spaces of rank one. Illinois J. Math. 21 (1977), 1 .. 41.

[6] HEJHAL, D.A.: The Selberg Trace Formula for PSL(2, R), Vol. I. Lect. Notes Math. 548 (1976), 1 - 516

[7] Hille, E.: Analytic Function Theory, Vol. I and II. Blaisdell: Ginn Comp 1962 and 1963.

[8] OlVER, F. W.: Asymptotics and Special Functions. New York: Academic Press 1974.

[9] SARNAK, P.: Determinants of Laplacians. Stanford preprint 1986.

[10] SCHUSTER, R.: The Selberg trace formula and a Poisson formula for the $p$-spectrum of compact hyperbolic space forms. Serdica, Bulg. math. publ. 12 (1986), $288-299$.

[11] SCHUSTER, R.: Spectral estimates for compact hyperbolic space forms and the Selberg zeta function for p-spactra. In preparation.

[12] SELBERG. A.: Harmonic analysis and discontinuous groups in weakly symmetric Riemannian spaces with applications to Dirichlet series. J. Indian Math. Soc. 20 (1956), $47-87$.

[13] TITCHMARSH, E.C.: The Theory of the Riemann Zeta Function. Oxford: Univ. Press 1951.

[14] VOROS, A.: Spectral functions, special functions and the Selberg zeta function. Comm. Math. Phys. 110 (1987), $439-465$

Received 24.09.1990; in revised form 22.11.1991

Dr. Reinhard Schuster

Institut fur Mathematik der Universität Leipzig

Augustusplatz 10

D (Ost) - 7010 Leipzig 